\title{
Micro Ion Source Program NA22 Plutonium Detection Portfolio Final Report
}

\author{
James E. Delmore
}

September 2010

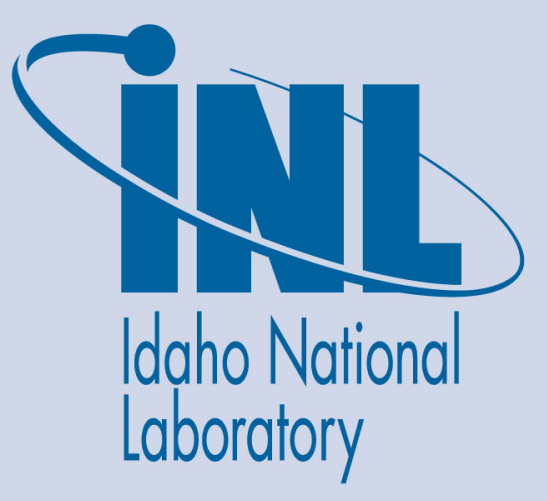

The INL is a U.S. Department of Energy National Laboratory operated by Battelle Energy Alliance 


\section{DISCLAIMER}

This information was prepared as an account of work sponsored by an agency of the U.S. Government. Neither the U.S. Government nor any agency thereof, nor any of their employees, makes any warranty, expressed or implied, or assumes any legal liability or responsibility for the accuracy, completeness, or usefulness, of any information, apparatus, product, or process disclosed, or represents that its use would not infringe privately owned rights. References herein to any specific commercial product, process, or service by trade name, trade mark, manufacturer, or otherwise, does not necessarily constitute or imply its endorsement, recommendation, or favoring by the U.S. Government or any agency thereof. The views and opinions of authors expressed herein do not necessarily state or reflect those of the U.S. Government or any agency thereof. 


\title{
Micro Ion Source Program NA22 Plutonium Detection Portfolio Final Report
}

\author{
James E. Delmore
}

September 2010

Idaho National Laboratory Idaho Falls, Idaho 83415

http://www.inl.gov

\author{
Prepared for the
}

U.S. Department of Energy

Office of National Nuclear Security Administration

Under DOE Idaho Operations Office

Contract DE-AC07-05ID14517 




\section{SUMMARY}

The purpose of the micro ion source program was to enhance the performance of thermal ionization mass spectrometry (TIMS) for various actinides and fission products. The proposal hypothesized that when ions are created at the ion optic center of the mass spectrometer, ion transmission is significantly increased and the resulting ion beam is more sharply focused. Computer modeling demonstrated this logic. In order to prove this hypothesis it was first necessary to understand the chemistry and physics governing the particular ion production process that concentrates the emission of ions into a small area. This has been achieved for uranium and technetium, as was shown in the original proposal and the improvement of both the beam transmission and sharpness of focus were proven. Significantly improved analytical methods have been developed for these two elements based upon this research. The iodine portion of the proposal turned out to be impractical due to volatility of iodine and its compounds. We knew this was a possibility prior to research and we proceeded anyway but did not succeed. Plutonium is a potential option, but is not quite up to the performance level of resin beads. Now, we more clearly understand the chemical and physical issues for plutonium, but have not yet translated this knowledge into improved analytical processes. The problems are that plutonium is considerably more difficult to convert to the required intermediate species, plutonium carbide, and the chemical method we developed that works with uranium functions only moderately well with plutonium. We are of the opinion that, with this knowledge, similar progress can be made with plutonium.

The breakthrough with uranium has come from development of what has been named the micro porous ion emitter (PIE). Many iterations of PIEs were developed and tested prior to settling on a particular formula. This formula is a $50 / 50$ blend of rhenium and platinum powders initially held together with melted hot glue and extruded into a very small diameter rope. This rope is sliced into very thin pieces that are positioned in the exact center of a rhenium mass spectrometer filament. The filament is then heated in a vacuum to first evaporate the hot glue and then to melt the platinum to alloy with the rhenium powder and filament. This mixture/formula results in a highly porous micro structure consisting of the rhenium/platinum alloy bonded to the rhenium filament. A water soluble ion exchange resin is wicked into the PIE and dried. The sample is then wicked into the PIE so that the element can absorbed onto the resin. The solution is allowed to sit in the PIE long enough for the element to absorb onto the ion exchange sites of the resin and then dried. The sample is then ready for analysis.

Three articles will be published in the International Journal of Mass Spectrometry; one is published, the second is accepted and the third has been submitted and is in review. The first appeared in the September 2009 issue and is entitled "Metal dicarbides as intermediate species in thermal ion formation mechanisms". This article describes the relationship between the ionization properties of the lanthanide and actinide elements prepared by the resin bead method and the heat of formation of the carbides. There is a striking relationship between these properties that is a strong indicator that carbides are the intermediate species responsible for ion formation. The second article is entitled "Porous Ion Emitters - A New Type of Thermal Ion Emitter." This article 
describes the preparation methods for the porous ion emitters and a preliminary assessment of applicability to uranium analysis. It is now available in the online version but is not yet in print. The third article has been submitted and is in review. It describes the extent to which the PIE limits ion emission to a confined region on the filament and how this benefits the analysis in terms of beam spreading. The extent of beam spreading then relates to sharpness of focusing, peak shape and ion transmission. Application to the analysis of trace amounts of ${ }^{233} \mathrm{U}$ and ${ }^{236} \mathrm{U}$ in a large excess of natural uranium are described.

An additional area of research that explains the behavior of uranium and plutonium on resin beads but has not yet advanced to the publication stage is what we refer to as the shell model. The shell model demonstrates that the plutonium nitrate anion cannot penetrate a significant distance into a resin bead. This explains why the method gives excellent sensitivity for very small samples but is not capable of being scaled in intensity. 


\section{CONTENTS}

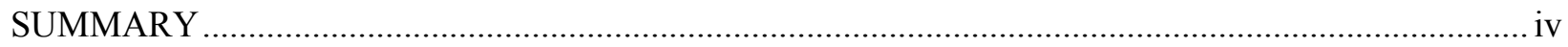

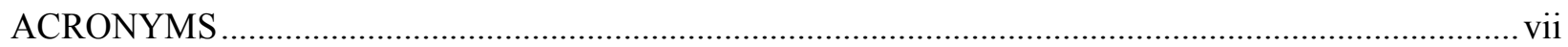

1. ACTINIDE AND LANTHANIDE ION FORMATION MECHANISMS ................................... 1

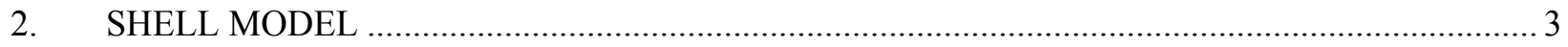

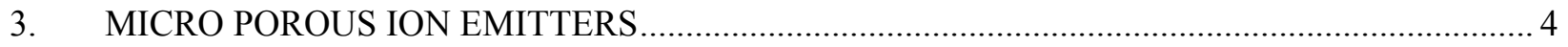

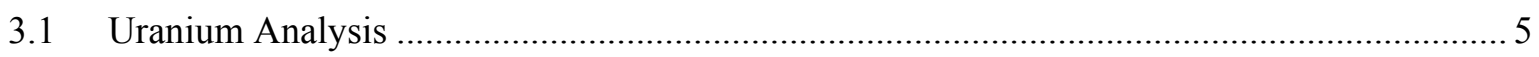

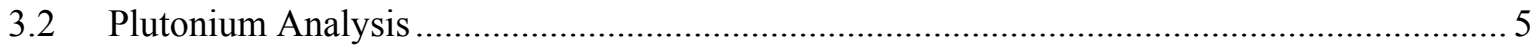

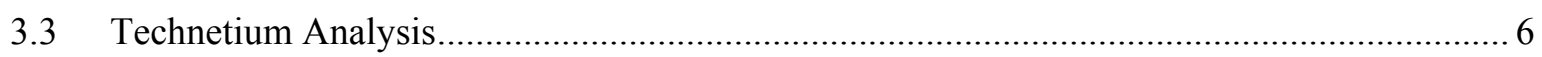

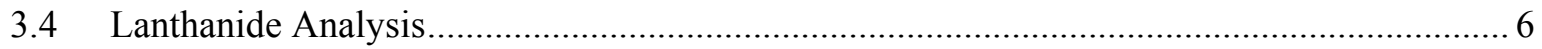

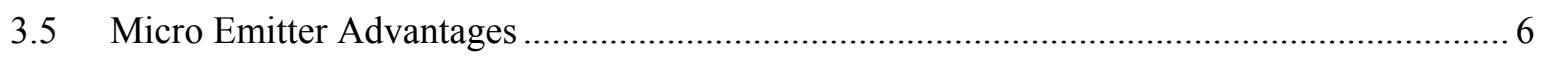

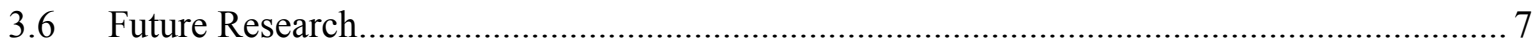

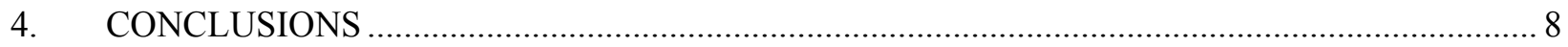

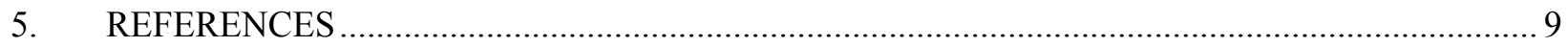

FIGURES

Figure 1. Observed lanthanide ion signal temperature vs. lanthanide carbide dissociation energy............. 1

Figure 2. Rhenium - platinum PIE sintered onto rhenium filament. ...................................................... 4 


\section{ACRONYMS}

PIE porous ion emitter

PNNL Pacific Northwest National Laboratory

TIMS thermal ionization mass spectrometry 


\section{Micro lon Source Program NA22 Plutonium Detection Portfolio Final Report}

\section{ACTINIDE AND LANTHANIDE ION FORMATION MECHANISMS}

Understanding the ion formation mechanisms responsible for the optimal ionization efficiencies for uranium and plutonium was a crucial first step toward the goal of concentrating ion formation from a large area to a much smaller area. A logical beginning in gaining this understanding would be to study trends across a series of elements with gradually changing properties. A problem with this approach was that the chemistry of the actinides does not have a gradual variation in chemical and physical properties because they are too abrupt. However, ion formation for all of the lanthanide elements and the more important actinides when analyzed in a carbon matrix appear to be very similar, if not identical. Therefore, a study was conducted that compared the ion formation temperatures and efficiencies for the lanthanides, uranium and plutonium. This resulted in the journal article mentioned in the Summary. The ion formation temperatures of these elements correspond very well with the temperature of formation of the carbides of the elements. This correlation is shown in Figure 1. In fact, the correlation is so strong that it leads to the conclusions that the carbides must be the intermediate species. A subsequent journal article by another laboratory provided confirmatory evidence to this conclusion. ${ }^{2}$

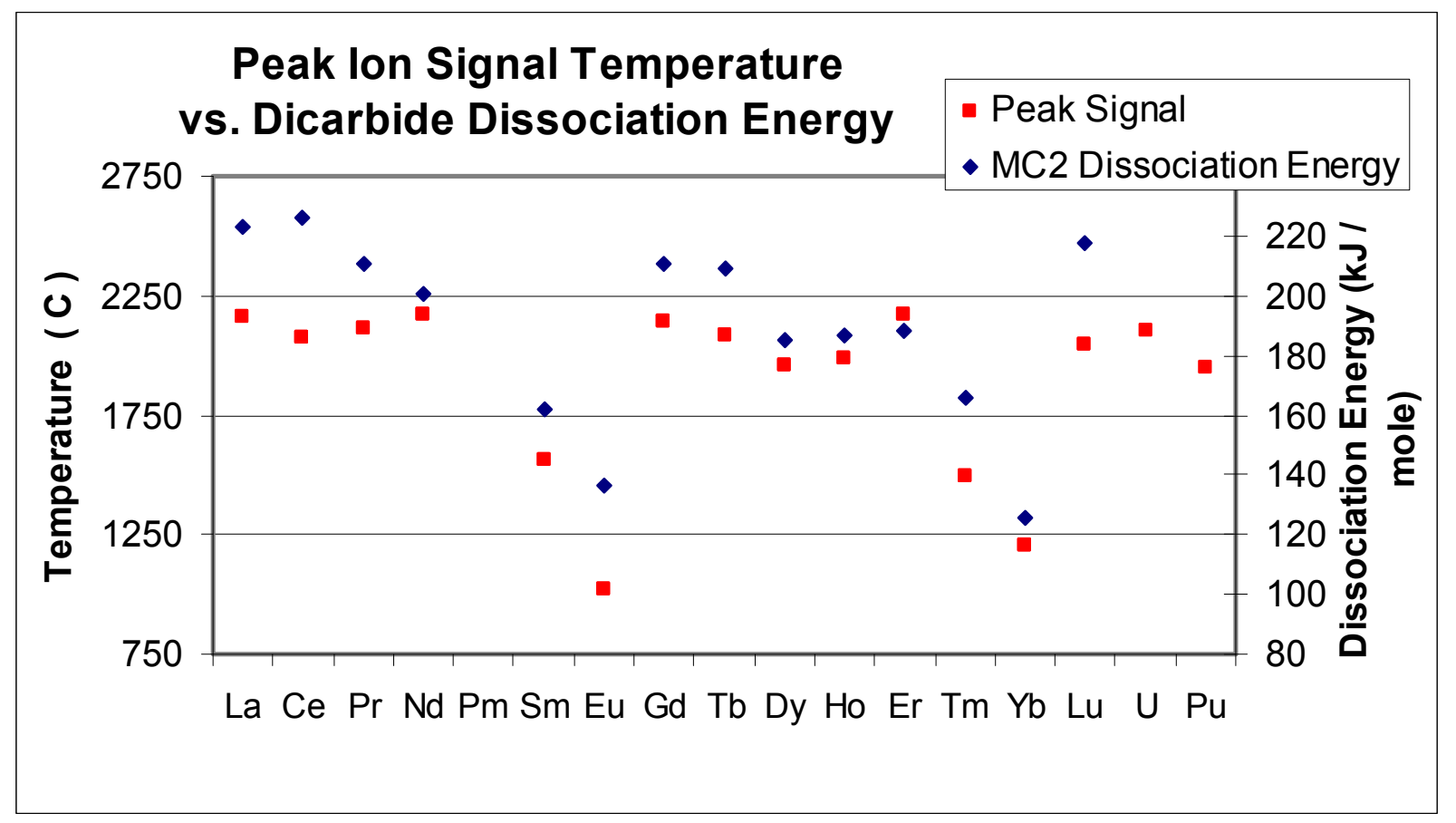

Figure 1. Observed lanthanide ion signal temperature vs. lanthanide carbide dissociation energy. 
Ion formation efficiencies were also studied. When progressing across the lanthanide series, the ionization efficiency dropped off significantly in a manner that had no correlation with the ionization potentials. A test was conducted where nearly identical amounts of both praseodymium and lutetium were loaded onto a resin bead, onto a porous ion emitter (PIE) and the relative ion formation efficiencies were measured. Depending upon the method for converting the elements to the carbide, there was between 5 and 100 times more praseodymium (a light lanthanide) ion intensity than lutetium (a heavy lanthanide) ion intensity. These two elements were chosen due to the fact that the ionization potentials are nearly identical, 5.464 versus $5.426 \mathrm{eV}(0.70 \%$ difference $)$ and they are on opposite ends of the lanthanide series. While praseodymium ion remained high regardless of the rigor of the carbide synthesis, the lutetium ion intensity changed by more than an order of magnitude. Such a strong swing in efficiencies with the preparation methods implies that this variation in ion intensity is due to lutetium being significantly more difficult to convert to the carbide than praseodymium. If there were a situation where lutetium carbide was fully or largely formed but was not efficient at decomposing to yield an ion, this ratio would be more constant. The only way to account for this huge swing in ion ratios is that the yield of lutetium carbide is poor to very poor. Literature surveys demonstrate that only the lighter lanthanides form nitrate complexes, and it is this anion complex that adsorbs onto the anion exchange resins. This fact explains the disparity in sensitivities. Apparently the ion needs to be chemically reacted with the carbon compound in order to be effectively converted to the carbide that produced the metal ion. This is a very important clue as to how to proceed. Unfortunately, the program ended before this relation was realized.

The same swing in efficiencies in traversing the lanthanides is observed in going from uranium to plutonium, only to a much lesser extent. When great care is taken in preparing plutonium on a resin bead, the ion formation efficiency is greater than for uranium. However, if shortcuts are taken in the preparation method, uranium efficiency is superior to plutonium. This leads to the conclusion that plutonium carbide is more difficult to synthesize than the uranium carbide. An unexplored possibility is that the adsorption efficiencies on resin beads may be slower. 


\section{SHELL MODEL}

We have developed a shell model of the resin bead analytical method. It has been known for many years that plutonium sensitivity increases significantly as the sample size decreases. Knowing this piece of historical information, we measured plutonium ion formation efficiency as a function of sample size. Sensitivity increased with decreasing sample size until a plateau was reached. At this point the sensitivity gain was about five. When this plateau was reached, sensitivity remained constant, although the ion intensity was close to the detection limits.

The observation of plutonium's sensitivity increasing in direct relation to sample size reduction was then used to develop a shell model of the resin bead. We calculated the number of cation sites in successively thinner outer shells of the resin bead. When the shell size reached nearly a single thickness of the ion exchange resin, the number of ion exchange sites was roughly correlated with the number of ions being produced at the edge of the plateau.

The correlation between ion exchange sites and number of ions being produced not only explains the shell phenomenon, but also reveals several limitations of the resin bead method. It has been known for a long time that the resin bead method gave excellent sensitivity for small samples, but that it could not be scaled up for larger samples. The shell model demonstrates why this is so. It also clarifies why impurities kill plutonium and uranium sensitivity.

The shell model also explains the limitations of attempting to measure ultra trace ${ }^{236} \mathrm{U}$ isotopes in a huge excess of natural uranium. The resin bead method has this inherent limitation that prevents it from being scaled up in intensity. Without the ability to scale ion emission, this difficult non-proliferation measurement is not possible.

A significantly larger database needs to be developed in order to confirm these conclusions and to publish the findings in a journal. Somewhere between six to twelve months of funding would be required, depending upon the level of studies on impurity effects on sample sensitivity: six months to develop a sufficient data base to quantify the shell model and another six months to demonstrate the effect of impurities. 


\section{MICRO POROUS ION EMITTERS}

Previous published studies (from Oak Ridge in the 1980's) demonstrated that, under tightly controlled conditions, the addition of rhenium metal powder on top of a resin bead sometimes gave a significant increase in plutonium sensitivity ${ }^{3}$. One downside to this technique is that reproducing results is highly problematic. After attempting this method and discovering just how temperamental this technique was, we initiated development of PIEs. PIEs are an attempt to gain reproducibility along with the significantly higher ionization efficiency that was obtained on a small percentage of analyses when adding rhenium powder to resin beads. This goal was achieved for uranium and a few lanthanide elements. Results for plutonium are still not as high as for resin beads, although we think we have identified the issue as not having a sufficiently robust technique for converting plutonium to the carbide inside the PIEs. These elements will be dealt with in subsequent sections, while this section will describe methods for producing PIEs.

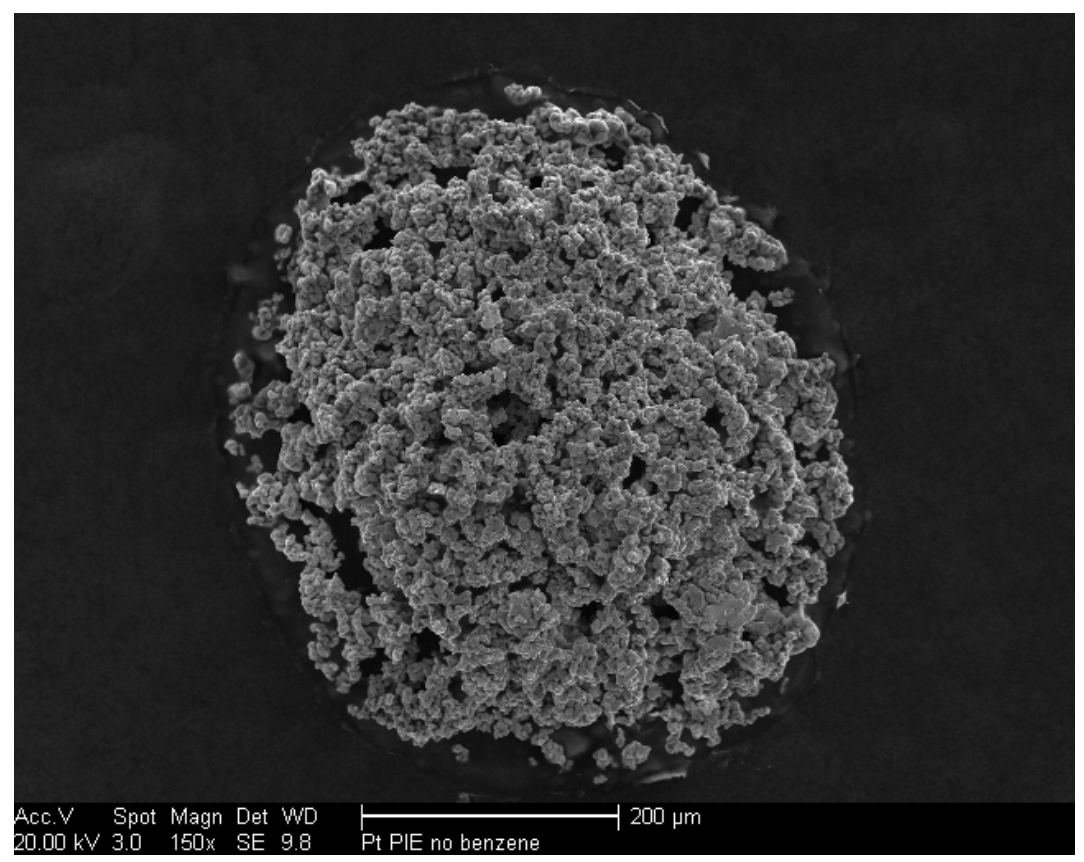

Figure 2. Rhenium - platinum PIE sintered onto rhenium filament.

A variety of techniques were tested for producing PIEs. These ranged from pure rhenium powder compacted into a small deposit to molten platinum to a blend of rhenium and platinum. The blends of rhenium and platinum were by far the best with approximately equal quantities giving the best results. Equal quantities of rhenium metal powder and platinum metal powder were blended, and then added to molten hot glue. PIEs work best when every effort was made to load as much metal powder into the hot glue as possible. This molten blend was sucked into a warm cutoff hypodermic needle and extruded into a very small, about $0.005 \mathrm{in}$., diameter rope. This was cooled to solidify the glue and the resulting rope was stored until ready for use. These were then cut into very thin slices and mounted at the center of a TIMS filament. These were heated to re-melt the glue to adhere this deposit to the filament. The filaments were then mounted in a vacuum chamber and slowly heated to $1900^{\circ} \mathrm{C}$. First, the glue evaporated and finally the temperature increased to a point in which a rhenium/platinum alloy formed. Rhenium is sufficiently refractory that it did not melt, but did alloy with the platinum. The resulting alloy coated the rhenium powder and the rhenium filament. The material was highly porous, had a small diameter covering about $1 / 3$ to $1 / 2$ of the width of the filament, and was tightly sintered to the center of the filament. This is pictured in Figure Two. This highly porous deposit cannot be picked off the filament. There are a variety 
of commercially available water soluble ion exchange resins, both anion and cation, that were wicked into these PIEs. These solutions required diluting with water in order to lower the viscosity to a point in which they could wick into these very small pores. They were dried to leave a deposit of the respective ion exchange resin coating the interior of the PIE. Small multiple drops of the solution containing the sample were wicked into the PIE and dried. These loaded filaments were treated the same as if they were resin beads. This consisted of mounting in a vacuum chamber, evacuating, slowly heating to $1400^{\circ} \mathrm{C}$ and introducing a low pressure of benzene. The filaments were then ready for analysis in the mass spectrometer.

A very important advantage of a PIE over a resin bead is that the PIE is not only sintered to itself but also to the filament. We have never had a PIE detach from a filament; in contrast to resin beads, which have had multiple instances of detachment. In fact, the PIE cannot even be physically removed; the sintering from alloy formation is too strong. In contrast, over the course of approximately 100 resin beads, approximately $20 \%$ detached from the filament and were lost during the benzene treatment step. This was determined by microscopic examination before and after benzene treatment. Another $5 \%$ were lost while in the instrument. Other labs claim that they never lose a bead, although that has not been our experience. It has been suggested that the problem we have encountered is related to the rate of heating and that we should reduce the heating rate. This may solve the lost resin bead problem, but one downside is that it lengthens a procedure that is already very time consuming.

\subsection{Uranium Analysis}

This subject is dealt with in the second journal article. The interested reader should refer to that article for details. Very briefly, when a certain heating pattern is followed, uranium ion formation efficiency as measured on the mass resolved detector of the mass spectrometer averaged $2 \%$. This compares to a reported average in the range of $0.5 \%$ for resin beads ${ }^{4}$. This factor of four is significant in itself, but an even more important advantage for a high value sample is that $25 \%$ of the samples may be lost with resin beads and this loss drops to zero for PIEs.

Another advantage of the PIE over the resin bead is mentioned in a following section on the advantages of micro emitters. This is the reduced tailing from a major isotope into a minor isotope. In turn, this relates to the ability to measure a minor isotope adjacent to a major isotope. Specifically, it helps in detection of the trace uranium isotopes at masses 232, 233 and 236 when looking for these isotopes in uranium stock that has allegedly never been in a reactor. This is an important non-proliferation analysis.

\subsection{Plutonium Analysis}

As already mentioned, after comparing plutonium analyses with PIEs and resin beads, the beads had a small sensitivity advantage. Plutonium on PIEs resulted in an average of $0.8 \%$ sample utilization efficiency ( $n=9,0.4-1.3 \%$ range). In contrast, plutonium on resin beads gave $1.2 \%$ sample utilization efficiency ( $\mathrm{n}=5,0.6-1.7 \%$ range). The beads had a 50\% sensitivity advantage over PIEs, although there was considerable overlap.

This raises the question as to why PIEs are more sensitive for uranium but less sensitive for plutonium. Also, since the ionization potential for plutonium $(6.06 \mathrm{eV})$ is more attractive for ion formation than for uranium $(6.19 \mathrm{eV})$, and with everything else being equal, plutonium should be more sensitive. It proves to be more sensitive than uranium by most ionization techniques. The answer was discovered when comparing $\mathrm{Pu} / \mathrm{U}$ ratios with $\mathrm{Pr} / \mathrm{Lu}$ ratios. A very long soaking time for a bead in a solution containing the element of interest $(\mathrm{U}, \mathrm{Pu}, \mathrm{Pr}$, and $\mathrm{Lu})$ allows the element to partially migrate into the interior of the organic matrix of the bead, although not very deeply as mentioned in the shell model. This allows the element a prolonged exposure to an organic matrix that is more likely to convert the element to 
the carbide. Since the PIE only has a thin coating of resin on the interior surfaces, there is less exposure to a carbon matrix during the pyrolysis step.

The conclusion from analyzing all of this information is that the bead is better at converting an element to the carbide, but once the carbide is formed, the PIE is more efficient at ion formation. In order for the PIE to become more sensitive for ionizing plutonium, it will be necessary to develop more robust technology for carbide formation.

\subsection{Technetium Analysis}

The elements technetium and rhenium have very similar chemistries, and we studied rhenium oxide ion formations under an Office of Science program. Using this as a basis, technetium oxide ion formation was demonstrated as a highly efficient mechanism for technetium analysis. We included technetium analysis as part of the proposal since it is an important fission product with non-proliferation possibilities and we felt that it would be a valuable tool for understanding micro emitters. This has indeed been verified. The studies were conducted jointly between the INL and Mark Engelmann at Pacific Northwest National Laboratory (PNNL). Mark developed a micro electrodeposition apparatus that made this project a success. This has been documented in a journal article. INL then took Mark's filaments with micro deposited technetium metal, conducted additional chemical steps, and analyzed them by mass spectrometry. The positioning experiments with technetium are included in the section on micro ion emitters.

\subsection{Lanthanide Analysis}

Lanthanide analysis was never a goal of this program, but in deciphering the carbide mechanism described in a previous section, it was obvious that the lighter lanthanide elements are ideal for analysis by either resin beads or PIEs. Sensitivity for neodymium analysis was consistently demonstrated to be in the range of $15 \%$. This is far higher than attainable by more standard methods. Since the samarium/neodymium parent/daughter pair is a very important geologic age dating tool, this has important implications for the age dating community. This might be a useful spin-off application.

However, the main programmatic advantage of the lanthanide analysis has been as the aid in understanding the ion formation mechanisms. First, the carbide is the critical intermediate chemical species, and second, some elements need a far more rigorous chemical synthetic method than others in order to produce the carbide. The second part derives from lutetium needing a more rigorous synthesis than praseodymium in order to produce the carbide, and by extrapolation, plutonium needing a more rigorous synthesis than uranium.

\subsection{Micro Emitter Advantages}

Ion optic modeling has demonstrated that ions produced at the ion optic center of the ion lens have higher transmission though the mass spectrometer by producing better resolution, peak shape and reduced tailing. This modeling is documented in the original proposal. This was experimentally demonstrated two ways.

First, with technetium ions originating only from a very small region, it was discovered that filament position was far more sensitive than when ions originated from a much larger area. The implication of this finding is that ions originating from the ion optic center of the lens are transmitted with far higher efficiency than when they are produced from a short distance away. When ions are produced across a broad cross section of the filament, a small movement of the filament only moves a different emitting area into the ion optic center. This greatly reduces the requirement for accurate filament placement. It also means that many ions are being discarded. When ion emission is concentrated into a small area, that area needs to be positioned very precisely. The upside of micro emission is that sensitivity is significantly 
increased and produces sharper focusing. The downside is the requirement for micro positioning of that emitting area.

Second, when the tailing of uranium ion beams produced with PIEs was compared to tailing from resin beads and triple filament sources, there was a significant improvement. The abundance sensitivity was about three times better for PIEs than for resin beads, and beads were about two times better than for the triple filament source. Implication of these measurements is that, even though PIEs and resin beads are comparable in size, the resin bead allows ion emission to spread over a larger area than the PIE. The resin bead has a smaller emission area than the triple filament ion source that presumably emits from the entire filament.

Comparing the migration of uranium to that of technetium is informative. Technetium ionization is a very low temperature process, less than $800^{\circ} \mathrm{C}$. Presumably, it does not readily migrate across the filament at this temperature. Uranium is ionized at approximately $1900^{\circ} \mathrm{C}$ and our experiments indicate that it does migrate at this temperature from resin beads. There does not appear to be migration across the filament when ionizing uranium from PIEs. This is attributed to the fact that there is a tortuous path that the element needs to traverse through the PIE in order to reach the filament. This was determined by the observation that the abundance sensitivity is improved by a factor of three for PIEs versus resin beads.

\subsection{Future Research}

A concept that ties all of this information together is that efficient ion formation has conversion to the carbide as a prerequisite. The question that then needs to be addressed is "what are the barriers to carbide formation?" The one concept that makes the most sense is that the element of interest needs to be chemically bonded to a carbonaceous material. Simply exposing the element to carbon is not sufficient. The resin bead method has accomplished this with bonding between the element of interest and the functional group on the styrene backbone. It appears that the limitations are related to the ability of the functional group to react with the element.

There are a number of possibilities for overcoming this problem of limited reactivity. The soluble ion exchange resin is one method, as it gave an order of magnitude sensitivity gain with PIES. Another possibility is to look at the many organic complexing reagents developed for the actinides. These complexing reagents may well be suitable for loading into PIES, and may be the breakthrough needed by the ultra trace plutonium analysis community. 


\section{CONCLUSIONS}

This program has clarified a number of important issues surrounding the issue of micro ion emitters for thermal ionization mass spectrometry. First, the computer generated ion optic models have been experimentally demonstrated to be accurate. Increased ion transmission and focusing sharpness have been borne out. Second, there is now a more accurate picture of the intermediate ion formation mechanisms for the actinides and lanthanides. The carbide is an essential intermediate species for these elements, and some elements are far more difficult to convert to the carbide than others. Third, a new type of ion emitter has been developed that is called the "micro porous ion emitter," or PIE for short. This is a development that holds promise for broad application in thermal ionization mass spectrometry. Fourth, there are useful new analytical methods for uranium and technetium.

In addition to these advances, there are two issues that require further study: First, it has been demonstrated that a very small micro emitter requires positioning accurate down to the micro level. This can be an issue with the current generation of filament positioning systems. The VG54 filament positioning system is just barely adequate for technetium. The PIEs used to date are very effective at limiting ion emission to the area of the PIE, that is demonstrated to be less than for a resin bead, but not as small an area as for technetium. Therefore, the VG filament positioning system is adequate for the current generation of PIEs but perhaps not for subsequent generation of smaller PIEs.

Second, it has been demonstrated that plutonium is intermediate in difficulty to convert to the carbide, the essential intermediate chemical species. A more robust synthesis of plutonium carbide would be a significant contribution to plutonium analysis. 


\section{REFERENCES}

1. Watrous, M. G.; Delmore, J. E. International Journal of Mass Spectrometry 2009, 286, 7.

2. Kraiem, M.; Mayer, K.; Gouder, T.; Seibert, A.; Wiss, T.; Thiele, H.; Hiernaut, J. P. International Journal of Mass Spectrometry 2010, 289, 108.

3. Smith, D. H.; Carter, J. A. International Journal of Mass Spectrometry and Ion Processes 1981, 40, 211.

4. Burger, S.; Riciputi, L. R.; Bostick, D. A.; Turgeon, S.; McBay, E. H.; Lavelle, M. International Journal of Mass Spectrometry 2009, 286, 70. 